\title{
FINITE PROJECTIONS IN TENSOR PRODUCT VON NEUMANN ALGEBRAS
}

BY

\author{
GEORGE A. ELLIOTT
}

\begin{abstract}
The work of Bures, Moore, Takenouchi, Hill and St $\phi$ rmer on the type classification of infinite tensor products of factors is extended to the nonfactor case.
\end{abstract}

1. Introduction. The basic result of this paper is that if a tensor product von Neumann algebra has a nonzero finite projection then it has a nonzero finite tensor product projection.

For tensor products of finitely many algebras this is a result of Sakai $[8$, Theorem 2]. For tensor products of infinitely many algebras, the first statement of this fact is due to Moore, who proved it for certain restricted tensor products of discrete factors [7, Lemma 5.2]. The restriction made by Moore was removed by Hill [5, Theorem 3.20] and by Takenouchi $[10, \S 3]$. In the present work the component algebras are not assumed to be discrete, and they are not assumed to be factors. If the centres are nonatomic a technical complication arises which is studied in the following section.

The basic result leads to a classification of tensor product von Neumann algebras with respect to type. In the factor case the contribution of the present paper is very small (see $\S 4.1$ below), although it does include simplifications of two arguments of Takenouchi. In the nonfactor case, it is of interest that the basic result, the existence of finite tensor product projections, plays a role in distinguishing discrete and continuous algebras, not just in distinguishing semifinite and purely infinite algebras.

2. A lemma on tensor products over commutative algebras.

2.1. Sublemma. Let $A$ and $B$ be $C^{*}$-algebras and let $Z_{1}$ and $Z_{2}$ be nonzero isomorphic sub-C*-algebras of the centres of $A$ and $B$. We shall identify $Z_{1}$ and $Z_{2}$ and denote them by $Z$. Denote by $A \otimes B$ the tensor product of the

Received by the editors July 22, 1972 and, in revised form, November 9, 1973.

AMS (MOS) subject classifications (1970). Primary 46L10, 46M05; Secondary 46L05.

Key words and phrases. von Neumann algebra, infinite tensor product, finite projection, tensor product projection.

Copyright $\odot$ 1975, American Mathematical Society 
involutive algebras $A$ and $B$. Denote by $S_{Z}$ the set of states of $A \otimes B$ of the form $\mu \otimes \nu$ where $\mu$ and $\nu$ are states of $A$ and $B$ such that $\mu \mid Z$ is equal to $\nu \mid Z$ and is a character of $Z$. Then the equation

$$
p_{Z}(x)^{2}=\sup \left\{\varphi\left(y^{*} x^{*} x y\right) \varphi\left(y^{*} y\right)^{-1} \mid y \in A \otimes B, \varphi \in S_{Z}\right\}
$$

defines a pre-C*-algebra seminorm $p_{Z}$ on $A \otimes B$ which is nonzero on $a \otimes b$ whenever $S_{Z}(a \otimes b) \neq 0$, and $p_{Z}$ is the smallest pre-C*-algebra seminorm on $A \otimes B$ with this property. Every state $\varphi \in S_{Z}$ has norm one with respect to $p_{Z}$.

Question. Must the kernel of $p_{Z}$ be the linear span of the set $\{a z \otimes b-$ $a \otimes z b \mid a \in A, z \in Z, b \in B\}$ ?

Proof. The assertion generalizes Theorem 2 of [11], and the proof is similar. We give an outline of the argument for the convenience of the reader.

It is enough to show that every $\varphi \in S_{Z}$ has norm one with respect to any given pre- $C^{*}$-algebra seminorm on $A \otimes B$ which is nonzero on $a \otimes b$ whenever $S_{Z}(a \otimes b) \neq 0$. By the Krein-Milman theorem it is enough to restrict attention to $\varphi \in S_{Z}$ of the form $\mu \otimes \nu$ where $\mu$ and $\nu$ are pure.

Suppose that there exist pure states $\mu_{0}$ and $\nu_{0}$ of $A$ and $B$ such that $\mu_{0} \mid Z$ equals $\nu_{0} \mid Z$ and is a character of $Z$ and such that $\mu_{0} \otimes \nu_{0}$ does not have norm one (with respect to a given pre- $C^{*}$-algebra seminorm on $A \otimes B$ with the specified kernel). We shall deduce a contradiction. There exist relatively open sets $U$ and $V$ of pure states of $A$ and $B$ with $\mu_{0} \in U$ and $\nu_{0} \in V$ such that no $\varphi \in U \otimes V$ has norm one. We may suppose that $U$ and $V$ are invariant under all automorphisms of $A$ and $B$ determined by unitary multipliers, for all such give rise to automorphisms of $A \otimes B$ fixing the given pre- $C^{*}$-algebra seminorm. Then by Lemma 8 of [4] there exist two-sided ideals $I$ of $A$ and $J$ of $B$ such that $U^{c}=I^{\perp}$ and $V^{c}=J^{\perp}$. Choose $a \in I^{+}$and $b \in J^{+}$such that $\mu_{0}(a) \neq 0$ and $\mu_{0}(b) \neq 0$. Then $\left(\mu_{0} \otimes \nu_{0}\right)(a \otimes b) \neq 0$, so $a \otimes b$ has nonzero seminorm.

At this point it is possible to deduce a contradiction if $A$ and $B$ are commutative. Indeed, every character of $A \otimes B$ of norm one must be of the form $\mu \otimes \nu$ with $\mu|Z=\nu| Z$, and is not in $U \otimes V$, whence either $\mu(a)=0$ or $\nu(b)=0$ and in any case $(\mu \otimes \nu)(a \otimes b)=0$. This, of course, contradicts the fact that $a \otimes b$ has nonzero seminorm.

In the general case there exist commutative sub-C*-algebras $A_{1}$ of $A$ containing $a$ and $Z$ and $B_{1}$ of $B$ containing $b$ and $Z$. Choose a pure state $\mu_{1}$ of $A_{1}$ such that $\mu_{1}\left|Z=\mu_{0}\right| Z$ and $\mu_{1}(a) \neq 0$ (this is possible because $\mu_{0}(a) \neq 0$ ), and also choose a pure state $\nu_{1}$ of $B_{1}$ such that $\nu_{1}\left|Z=\nu_{0}\right| Z$ and $\nu_{1}(b) \neq 0$. Then by the conclusion in the commutative case, proved in the preceding paragraph, $\mu_{1} \otimes$ $\nu_{1}$ is a state of norm one of $A_{1} \otimes B_{1}$. It is, of course, pure. Extend $\mu_{1} \otimes \nu_{1}$ to 
a pure state $\theta$ of $A \otimes B_{1}$ of norm one. Then $\theta$ has norm one with respect to the largest pre-C*-algebra norm on $A \otimes B_{1}$. Hence by Lemma 4 of [11] (which it is not necessary to generalize), $\theta=\mu_{2} \otimes \nu_{1}$ with $\mu_{2}$ pure. Extend $\mu_{2} \otimes \nu_{1}$ to a pure state $\theta^{\prime}$ of norm one of $A \otimes B$. Then $\theta^{\prime}$ has norm one with respect to the largest pre- $C^{*}$-algebra norm on $A \otimes B$, and hence, by Lemma 4 of [11], $\theta^{\prime}=$ $\mu_{2} \otimes \nu_{2}$ with $\nu_{2}$ pure. But $\mu_{2} \in U$ and $\nu_{2} \in V$ since $\mu_{2}(a) \neq 0$ and $\nu_{2}(b) \neq 0$; hence, by the construction of $U$ and $V, \mu_{2} \otimes \nu_{2}$ does not have norm one. This, of course, contradicts the construction of $\mu_{2} \otimes \nu_{2}=\theta^{\prime}$ to have norm one.

2.2. Lemma. Let $A$ and $B$ be commuting $C^{*}$-algebras of operators with nonzero intersection $Z$. Suppose that the kernel of the canonical map $\beta$ from the tensor product $A \otimes B$ of the algebras $A$ and $B$ onto the algebra $R$ generated by $A$ and $B$ is generated by $\{a z \otimes b-a \otimes z b \mid a \in A, z \in Z, b \in B\}$, so that if $\pi: A \rightarrow Z$ and $\rho: B \rightarrow Z$ are $Z$-linear there exists a unique $Z$-linear map $\pi \otimes_{Z} \rho:$ $R \rightarrow Z$ such that $\left(\pi \otimes_{Z} \rho\right)(a b)=\pi(a) \rho(b), a \in A, b \in B$. Then if $\pi$ and $\rho$ are projections of norm one, so is $\pi \otimes_{Z} \rho$.

Proof. It is enough to show that $\pi \otimes_{Z} \rho$ has norm one. To show this it is enough to show that $\gamma \circ\left(\pi \otimes_{Z} \rho\right)$ has norm one for each character $\gamma$ of $Z$. But if $\gamma$ is a character of $Z, \gamma \circ\left(\pi \otimes_{Z} \rho\right) \circ \beta=\gamma \circ \pi \otimes \gamma \circ \rho$, so by $2.1 \gamma \circ$ $\left(\pi \otimes_{Z} \rho\right) \circ \beta$ has norm one on $A \otimes B$ with respect to the preimage by $\beta$ of the operator norm on $R$. This says that $\gamma \circ\left(\pi \otimes_{Z} \rho\right)$ has norm one.

2.3. Problem. Let $A$ and $B$ be $C^{*}$-algebras, and let $C$ be a $C^{*}$-algebra isomorphic to sub- $C^{*}$-algebras $C_{1}$ of $A$ and $C_{2}$ of $B$, which we shall denote by $C$. Denote by $A \otimes_{C} B$ the quotient of the tensor product $A \otimes B$ of the involutive algebras $A$ and $B$ by the two-sided ideal generated by the set $\{a c \otimes b-a \otimes c b \mid$ $a \in A, c \in C, b \in B$, so that if $\pi: A \rightarrow C$ is a right $C$-linear map and $\rho: B \rightarrow$ $C$ is a left $C$-linear map then there exists a unique complex-linear map $\pi \otimes_{C} \rho$ : $A \otimes_{C} B \rightarrow C$ such that $\left(\pi \otimes_{C} \rho\right)\left(a \otimes_{C} b\right)=\pi(a) \rho(b)$. It is not difficult to show that $C$ is isomorphic to $C \otimes_{C} C$ (by the map $c_{1} \otimes_{C} c_{2} \rightarrow c_{1} c_{2}$ ), so that if $\pi$ and $\rho$ are projections so is $\pi \otimes_{C} \rho$. If $\pi$ and $\rho$ are projections of norm one, must $\pi \otimes_{C} \rho$ also be a projection of norm one, with respect to any pre-C*-algebra norm on $A \otimes_{C} B$ ?

3. The existence of finite tensor product projections.

3.1. Notation and preliminaries. Using the notation of Bures [2] for tensor products, we shall write $A=\bigotimes\left(A_{i}, \mu_{i}\right)$ to mean that $\mu_{i}$ is a normal state of the von Neumann algebra $A_{i}$ for every index $i$ and that $A$ is the tensor product of the family of von Neumann algebras $\left(A_{i}\right)$ with respect to the family of states $\left(\mu_{i}\right)$. This does not specify the Hilbert space on which $A$ acts, but if each $\mu_{i}$ is faithful 
then the cyclic representation of $A$ defined by the tensor product state $\otimes \mu_{i}$ is faithful.

Although it is not necessary to refer to the Hilbert space on which each $A_{i}$ acts, we shall do so in order to apply 2.2 conveniently. (This lemma could itself have been formulated more abstractly, but the present formulation seems the least cumbersome.) We shall assume that the action of $A_{i}$ on the Hilbert space $H_{i}$ is standard, in the sense that there exists an involutory antiunitary $J_{i}$ in $H_{i}$ with the properties that $J_{i} A_{i} J_{i}=A_{i}^{\prime}$ and $J_{i} z J_{i}=z^{*}$ for each $z \in Z_{i}=A_{i} \cap A_{i}^{\prime}$. The existence in general of such an action was established only relatively recently by work of Tomita (see [12]), but we shall actually need to refer to $J_{i}$ only in the case that $A_{i}$ is finite (and even countably decomposable, so that there exists a faithful finite normal trace on $A_{i}$ ), in which case the existence of $J_{i}$ is classical. We shall denote $J_{i} x J_{i}$ by $j(x), x \in A_{i}$.

If for each $i$ a projection $e_{i}$ in $A_{i}$ is given, we shall denote by $\bigotimes e_{i}$ the infimum in $\otimes\left(A_{i}, \mu_{i}\right)$ of the finite products of the projections $e_{i} \otimes 1$. We shall use the easily established fact that a sufficient condition for $\otimes e_{i}$ to be nonzero is that the product of scalars $\Pi \mu_{i}\left(e_{i}\right)$ be nonzero. The infinite product is of course defined to be the limit of the finite products, or, equivalently, since all the factors belong to the interval $[0,1]$, the infimum of the finite products. A characterization of the nonvanishing of the product $\Pi \lambda_{i}$ of nonzero $\lambda_{i}$ in the interval $[0,1]$ that we shall use is the convergence of the sum $\Sigma\left(1-\lambda_{i}\right)$. (This characterization follows from the inequality $(1-\lambda) \leqslant-\log \lambda \leqslant(2 \log 2)(1-\lambda), \lambda \in[1 / 2,1]$.

If $A_{i}$ is finite and $\mu_{i}$ is faithful (a situation which will predominate), then there exists a unique trace on $A_{i}$ which coincides with $\mu_{i}$ on the centre $Z_{i}$ of $A_{i}$. We shall denote this trace by $\tau_{i}$; necessarily $\tau_{i}$ is faithful, normal and finite. There exists a unique positive operator $h_{i}$ affiliated with $A_{i}$ and of finite trace (with respect to $\tau_{i}$ ) such that $\mu_{i}=\tau_{i} h_{i}$ (i.e. $\mu_{i}(x)=\tau_{i}\left(h_{i} x\right)$ for all $\left.x \in A_{i}\right) ; h_{i}$ is called the Radon-Nikodým derivative of $\mu_{i}$ with respect to $\tau_{i}$. The case that $h_{i}$ is bounded and invertible will be dominant in the considerations that follow.

If $\mu_{i}$ is a faithful normal state of $A_{i}$ then (whether $A_{i}$ is finite or not) there exists a unique projection $\pi_{i}$ of norm one from $A_{i}$ onto $Z_{i}$, the centre of $A_{i}$, such that $\mu_{i}=\mu_{i} \circ \pi_{i}$. This is easily established, using the commutative case of the preceding Radon-Nikodým theorem. Necessarily $\pi_{i}$ is normal and is $Z_{i}$-linear.

The preceding notation will be used without comment in what follows. We shall also use the notation introduced in 2.2, in the case $A=A_{i}$ and $B=A_{i}^{\prime}$.

An additional notation that we shall use (following Moore [6]) is $|x|_{c}$, where $x$ is an operator and $c>0$ is real, to denote $\inf \{|x|, c\}$.

3.2. Theorem. Let $A=\bigotimes\left(A_{i}, \mu_{i}\right)$. Suppose that there exists a nonzero finite projection in $A$. Then there exists for each $i$ a nonzero projection $e_{i} \in A_{i}$ such that $\otimes e_{i}$ is nonzero and finite. 
Proof. By the result of Sakai referred to in the second paragraph of 1 , if $e$ is a purely infinite projection of $A_{i}$ then $e \otimes 1$ is a purely infinite projection of $A$. Hence the largest semifinite projection in $A$ is contained in the tensor product of the largest semifinite projections of the algebras $A_{i}$. (The complement of this projection is a sum of purely infinite central projections and is therefore purely infinite.) It is thus seen to be sufficient to consider the case that each $A_{i}$ is semifinite.

If each $A_{i}$ is semifinite then the largest semifinite projection in $A$ is, by Sakai's result and the fact that the tensor product of two semifinite von Neumann algebras is semifinite, contained for each finite set of indices $F$ in $\left\{A_{i} \otimes 1 \mid i \notin F\right\}^{\prime \prime}$. Since the intersection of these algebras over all finite sets $F$ is the scalars, $A$ is in this case either semifinite or purely infinite. Since by hypothesis $A$ has a nonzero finite projection, the assumption that each $A_{i}$ is semifinite, which we shall make henceforth, entails that $A$ also is semifinite.

If for each $i$ a nonzero projection $f_{i}$ in $A_{i}$ is specified, with $\mu_{i}\left(f_{i}\right) \neq 0$, and if $\otimes f_{i}$ is nonzero, then it is sufficient to prove the conclusion of the theorem after the replacement of each $A_{i}$ by $f_{i} A_{i} f_{i}$, of each $\mu_{i}$ by $\mu_{i}\left(f_{i}\right)^{-1} \mu_{i} \mid f_{i} A_{i} f_{i}$, and of $A$ by $\left(\otimes f_{i}\right) A\left(\otimes f_{i}\right)$. Since such projections $f_{i}$ can be chosen so that each $f_{i} A_{i} f_{i}$ is finite, each $\mu_{i} \mid f_{i} A_{i} f_{i}$ is faithful, and each $h_{i}$ is bounded and invertible, we see that it is enough to prove the theorem in the case that each $A_{i}$ is finite, each $\mu_{i}$ is faithful, and each $h_{i}$ is bounded and invertible.

In this case, the theorem follows from $3.3,3.5,3.6,3.7$ (here projections $e_{i}$ are constructed with $\otimes e_{i} \neq 0$ ), and 3.8.

3.3. Lemma. Let $A=\bigotimes\left(A_{i}, \mu_{i}\right)$. Assume that each $A_{i}$ is finite and that each $\mu_{i}$ is faithful. Then if $A$ is semifinite,

$$
\sum \mu_{i}\left(1-\left|\pi_{i}\left(h_{i}^{i t}\right)\right|\right)<\infty \text { for all real } t .
$$

Proof (Moore [7]). Fix a faithful semifinite normal trace $\tau$ on $A$, set $\otimes \mu_{i}=\mu$, and denote by $h$ the Radon-Nikodým derivative of $\mu$ with respect to $\tau$ (so that $\mu=\tau h$ ). For each finite subset $F$ of indices set $\bigotimes_{i \notin F}\left(A_{i}, \mu_{i}\right)=A_{F}$ and $\bigotimes_{i \notin F} \mu_{i}=\mu_{F}$, and denote by $\pi_{F}$ the unique projection of norm one onto the centre of $A_{F}$ such that $\mu_{F}=\mu_{F} \circ \pi_{F}$. We have $A_{\varnothing}=A$ and $\mu_{\varnothing}=\mu$, and we may write $\pi_{\phi}=\pi$. For each $F$ fix a faithful semifinite normal trace $\tau_{F}$ on $A_{F}$. (We may choose $\tau_{\varnothing}$ to be $\tau$, but if $F \neq \varnothing$ then the restriction of $\tau$ to $A_{F}$ may fail to be semifinite.) Denote by $h_{F}$ the Radon-Nikodým derivative of $\mu_{F}$ with respect to $\tau_{F}$ (so that $\mu_{F}=\tau_{F} h_{F}$ ). Then by the essential uniqueness of the trace on $A$, for each $F$ there exists an operator $z_{F}$ affiliated with the centre of $A$ such that $\left(\otimes_{i \in F} h_{i}\right) \otimes h_{F}=z_{F} h$.

Hence, for each real $t$, in succession: 


$$
\begin{gathered}
\left(\bigotimes_{i \in F} h_{i}^{i t}\right) \otimes h_{F}^{i t}=z_{F}^{i t} h^{i t} ; \\
\left(\bigotimes_{i \in F} \pi_{i}\left(h_{i}^{i t}\right)\right) \otimes \pi_{F}\left(h_{F}^{i t}\right)=\pi\left(z_{F}^{i t} h^{i t}\right) ; \\
\left(\bigotimes_{i \in F}\left|\pi_{i}\left(h_{i}^{i t}\right)\right|\right) \otimes\left|\pi_{F}\left(h_{F}^{i t}\right)\right|=\left|\pi\left(h^{i t}\right)\right| ; \\
\left(\prod_{i \in F} \mu_{i}\left(\left|\pi_{i}\left(h_{i}^{i t}\right)\right|\right)\right) \mu_{F}\left(\left|\pi_{F}\left(h_{F}^{i t}\right)\right|\right)=\mu\left(\left|\pi\left(h^{i t}\right)\right|\right) ; \\
\prod_{i \in F} \mu_{i}\left(\left|\pi_{i}\left(h_{i}^{i t}\right)\right|\right) \geqslant \mu\left(\left|\pi\left(h^{i t}\right)\right|\right) .
\end{gathered}
$$

Therefore the sum $\Sigma \mu_{i}\left(1-\left|\pi_{i}\left(h_{i}^{i t}\right)\right|\right)$ is finite at least for all $t$ such that $\mu\left(\left|\pi\left(h^{i t}\right)\right|\right)$ $\neq 0$, and since $\pi$ is ultrastrongly continuous this means at least for all $t$ in an interval about 0 . This is all that we shall need, but since for any $t$ there exists a finite set $K$ such that $\pi_{K}\left(h_{K}^{i t}\right) \neq 0$ (there exists $u \in\left(\bigotimes_{i \in K} A_{i}\right) \otimes 1$ for some finite $K$ such that $\pi\left(u h^{i t}\right) \neq 0$, and then $\pi_{K}\left(h_{K}^{i t}\right) \neq 0$ because $h^{i t}$ is a central unitary multiple of $\left.\left(\otimes_{i \in K} h_{i}^{i t}\right) \otimes h_{K}^{i t}\right)$, the sum is finite for all $t$.

3.4. REMARK. The converse implication to that of 3.3 is also true, as is shown by the sequel.

If the predual of $A=\bigotimes\left(A_{i}, \mu_{i}\right)$ is separable, this may also be shown as in $\left[9\right.$, p. 814]. First, observe that for each $t$ the condition $\Sigma \mu_{i}\left(1-\left|\pi_{i}\left(h_{i}^{i t}\right)\right|\right)<\infty$ is equivalent to the innerness of the modular automorphism $\sigma_{t}^{\mu}$ defined in [12], where $\mu=\otimes \mu_{i}$ (cf. the paragraph which follows). If $\sigma_{t}^{\mu}$ is inner for every $t$, then by Theorem 0.1 of [6] the one-parameter group $t \mapsto \sigma_{t}^{\mu}$ is inner, in the sense of [12], whence by Theorem 14.1 of [12], $A$ is semifinite. (Note that this method is not applicable if the predual of $A$ is not separable; a purely infinite factor with only inner modular automorphisms is constructed in 1.5 of [3] .)

It is perhaps worth remarking that for arbitrary unitaries $u_{i} \in A_{i}$, the condition $\Sigma \mu_{i}\left(1-\left|\pi_{i}\left(u_{i}\right)\right|\right)<\infty$ is equivalent to the existence (in $\otimes\left(A_{i}, \mu_{i}\right)$ ) of the tensor product $\otimes v_{i}^{*} u_{i}$, where $v_{i}$ is (any) unitary in the centre of $A_{i}$ such that $\pi_{i}\left(u_{i}\right)=v_{i}\left|\pi_{i}\left(u_{i}\right)\right|$. Existence in $\otimes\left(A_{i}, \mu_{i}\right)$ of $\otimes v_{i}^{*} u_{i}$ of course means strong convergence of the finite products of the elements $v_{i}^{*} u_{i} \otimes 1$.

3.5. Lemma. Let $A=\bigotimes\left(A_{i}, \mu_{i}\right)$. Assume that each $A_{i}$ is finite and that each $\mu_{i}$ is faithful. Then

$$
\sum \mu_{i}\left(1-\left|\pi_{i}\left(h_{i}^{i t}\right)\right|\right)<\infty \Longleftrightarrow \sum \mu_{i}\left(\pi_{i} \otimes_{Z_{i}} j \circ \pi_{i} \circ j\left(\left|h_{i}^{i t} j\left(h_{i}^{i \dagger}\right)-1\right|^{2}\right)\right)<\infty .
$$

Proof (cf. [9, p. 816]). First, it follows from the inequalities

$$
1-\lambda \leqslant 1-\lambda^{2}=(1-\lambda)(1+\lambda) \leqslant 2(1-\lambda), \quad 0 \leqslant \lambda \leqslant 1,
$$


that

$$
\sum \mu_{i}\left(1-\left|\pi_{i}\left(h_{i}^{i t}\right)\right|\right)<\infty \Longleftrightarrow \sum \mu_{i}\left(1-\left|\pi_{i}\left(h_{i}^{i t}\right)\right|^{2}\right)<\infty
$$

Then, for each $i$,

$$
\begin{aligned}
\pi_{i} \otimes_{Z_{i}} j \circ \pi_{i} \circ j\left(\left|h_{i}^{i t} j\left(h_{i}^{i t}\right)-1\right|^{2}\right) & =\pi_{i} \otimes_{Z_{i}} j \circ \pi_{i} \circ j\left(2-h_{i}^{i t} j\left(h_{i}^{i t}\right)-h_{i}^{-i t} j\left(h_{i}^{-i t}\right)\right) \\
& =2-\pi_{i}\left(h_{i}^{i t}\right) j \circ \pi_{i}\left(h_{i}^{i t}\right)-\pi_{i}\left(h_{i}^{-i t}\right) j \circ \pi_{i}\left(h_{i}^{-i t}\right) \\
& =2-\pi_{i}\left(h_{i}^{i t}\right) \pi_{i}\left(h_{i}^{-i t}\right)-\pi_{i}\left(h_{i}^{-i t}\right) \pi_{i}\left(h_{i}^{i t}\right) \\
& =2\left(1-\left|\pi_{i}\left(h_{i}^{i t}\right)\right|^{2}\right) .
\end{aligned}
$$

3.6. Lemma. Let $A=\bigotimes\left(A_{i}, \mu_{i}\right)$. Assume that each $A_{i}$ is finite and that each $\mu_{i}$ is faithful. Assume that each $h_{i}$ is bounded and invertible. Then, for each real $c>0$,

$$
\begin{aligned}
& \sum \mu_{i}\left(\pi_{i} \otimes_{z_{i}} \circ \circ \pi_{i} \circ j\left(\left|h_{i}^{i t} j\left(h_{i}^{i t}\right)-1\right|^{2}\right)\right)<\infty \\
& \Longleftrightarrow \sum \mu_{i}\left(\pi_{i} \otimes_{z_{i}} j \circ \pi_{i} \circ j\left(\left|h_{i} j\left(h_{i}^{-1}\right)-1\right|_{c}^{2}\right)\right)<\infty .
\end{aligned}
$$

Proof (Moore [7]). To simplify notation we restate the lemma in a more abstract form: If for each $i$ a positive form $\varphi_{i}$ is given on the $C^{*}$-algebra $B_{i}$ and an element $x_{i}=x_{i}^{*}$ of $B_{i}$ is given, then, for each real $c>0$,

$$
\sum \varphi_{i}\left(\left|1-\exp i t x_{i}\right|^{2}\right)<\infty \text { for all real } t \Longleftrightarrow \sum \varphi_{i}\left(11-\left.\exp x_{i}\right|_{c} ^{2}\right)<\infty \text {. }
$$

Before proving this equivalence, we note that by the inequality

$$
(\log 2)|1-\exp \lambda| \leqslant|\lambda| \leqslant(2 \log 2)|1-\exp \lambda| \text { for }|1-\exp \lambda| \leqslant 1 / 2,
$$

the convergence of the sum $\Sigma \varphi_{i}\left(11-\left.\exp x_{i}\right|_{c} ^{2}\right)$ is, for $0<c \leqslant 1 / 2$, equivalent to the convergence of the sum $\Sigma \varphi_{i}\left(\left|x_{i}\right|_{c}^{2}\right)$. Moreover, if either of these sums is convergent for a single real $c>0$, it is convergent for all such $c$. So it is enough to prove that

$$
\sum \varphi_{i}\left(11-\left.\exp i t x_{i}\right|^{2}\right)<\infty \text { for all real } t \Longleftrightarrow \sum \varphi_{i}\left(\left|x_{i}\right|_{c}^{2}\right)<\infty \text {. }
$$

By writing each $x_{i}$ as $x_{i}^{\prime}+x_{i}^{\prime \prime}$ where $x_{i}^{\prime} \in \epsilon_{i}^{\prime} B_{i} e_{i}^{\prime}$ and $x_{i}^{\prime \prime} \in e_{i}^{\prime \prime} B_{i} e_{i}^{\prime \prime}$ with $e_{i}^{\prime}$ and $e_{i}^{\prime \prime}$ orthogonal projections in the bidual of $B_{i}$ and $\left|x_{i}^{\prime}\right| \geqslant c e_{i}^{i},\left|x_{i}^{\prime \prime}\right| \leqslant c$, and replacing $\varphi_{i}$ by $\varphi_{i}^{\prime}+\varphi_{i}^{\prime \prime}$ where $\varphi_{i}^{\prime}=e_{i}^{\prime} \varphi_{i} e_{i}^{\prime}$ and $\varphi^{\prime \prime}=e_{i}^{\prime \prime} \varphi_{i} e_{i}^{\prime \prime}$, we may suppose that each $x_{i}$ satisfies either $\left|x_{i}\right| \geqslant c$ or $\left|x_{i}\right| \leqslant c$.

We have for each real $t$, 


$$
\left|1-\exp i t x_{i}\right|^{2}=2\left(\left(t x_{i}\right)^{2} / 2 !-\left(t x_{i}\right)^{4} / 4 !+\cdots\right),
$$

so if $t$ is small enough that $t^{2} x_{i}^{2} \leqslant 1$, then

$$
\left(t x_{i}\right)^{2}-\left(t x_{i}\right)^{4} / 12 \leqslant\left|1-\exp i t x_{i}\right|^{2} \leqslant\left(t x_{i}\right)^{2} .
$$

$\operatorname{Ad} \Rightarrow$. We have $1-t^{2} x_{i}^{2} / 12 \geqslant 1-t^{2} c^{2} / 12$ if $\left|x_{i}\right| \leqslant c$, so if $t$ is small enough that $1-t^{2} c^{2} / 12>0$, then

$$
\sum_{\left|x_{i}\right|<c} \varphi_{i}\left(\left|1-\exp i t x_{i}\right|^{2}\right)<\infty \Rightarrow \sum_{\left|x_{i}\right|<c} \varphi_{i}\left(\left|x_{i}\right|_{c}^{2}\right)<\infty .
$$

We must show that

$$
\sum_{\left|x_{i}\right| \geq c} \varphi_{i}\left(\left|1-\exp i t x_{i}\right|^{2}\right)<\infty \text { for all real } t \Rightarrow \sum_{\left|x_{i}\right|<c}\left\|\varphi_{i}\right\|<\infty .
$$

Since the map $t \mapsto \Sigma_{\left|x_{i}\right| \geqslant c} \varphi_{i}\left(\left|1-\exp i t x_{i}\right|^{2}\right)$ is lower semicontinuous, if it is everywhere finite it must be bounded on some interval. Denote by $I$ an interval of nonzero length $|\eta|$ such that for some $M>0$,

$$
\sum_{\left|x_{i}\right|>c} \varphi_{i}\left(\left|1-\exp i t x_{i}\right|^{2}\right) \leqslant M \quad \text { for all } t \in I .
$$

Then

$$
\sum_{\left|x_{i}\right| \geq c} \varphi_{i}\left(\int_{I}\left|1-\exp i t x_{i}\right|^{2} d t\right) \leqslant M|I|
$$

It is now enough to show that for some $m>0$,

$$
m \leqslant \int_{I}\left|1-\exp i t x_{i}\right|^{2} d t \quad \text { for all } i \text { with }\left|x_{i}\right| \geqslant c .
$$

It is enough to show that for some $m>0$,

$$
m \leqslant \int_{I}|1-\exp i t s|^{2} d t \text { for all real } s \text { with }|s| \geqslant c .
$$

This holds since the function $s \mapsto \int_{I}|1-\exp i t s|^{2} d t$ is continuous, nonzero, and by the Riemann-Lebesgue lemma has limit $2|I|$ as $s \rightarrow \infty$.

$\mathrm{Ad} \Leftarrow$. (This implication will not be needed.) Fix $t$. Choose $c>0$ so that $t^{2} c^{2} \leqslant 1$. From the inequality immediately preceding the proof of $\Rightarrow$,

$$
\sum_{\left|x_{i}\right|<c} \varphi_{i}\left(\left|x_{i}\right|_{c}^{2}\right)<\infty \Rightarrow \sum_{\left|x_{i}\right|<c} \varphi_{i}\left(11-\left.\exp i t x_{i}\right|^{2}\right)<\infty .
$$

Also (independently of the choice of $c$ ),

$$
\sum_{\left|x_{i}\right| \geq c} \varphi_{i}\left(\left|x_{i}\right|_{c}^{2}\right)<\infty \Rightarrow \sum_{\left|x_{i}\right| \geqslant c}\left\|\varphi_{i}\right\|<\infty \Rightarrow \sum_{\left|x_{i}\right| \geq c} \varphi_{i}\left(\left|1-\exp i t x_{i}\right|^{2}\right)<\infty .
$$


3.7. Lemma. Let $A=\bigotimes\left(A_{i}, \mu_{i}\right)$. Assume that the hypotheses of 3.6 are satisfied. Suppose that the second of the equivalent conditions of 3.6 is satisfied. Then it is possible to choose for each $i$ a projection $e_{i}$ in the von Neumann algebra generated by $h_{i}$ such that $e_{i} h_{i} j\left(e_{i} h_{i}^{-1}\right) \leqslant 8$, in such a way that $\Sigma \mu_{i}\left(1-e_{i}\right)<\infty$.

Proof. For each $n \in Z$ denote by $e_{i}^{n}$ the spectral projection of $h_{i}$ corresponding to the half-open interval $\left[2^{n}, 2^{n+1}[\right.$.

For each $i$,

$$
\sum_{|m-n|>1} e_{i}^{m} j\left(e_{i}^{n}\right) \leqslant\left|h_{i} j\left(h_{i}^{-1}\right)-1\right|_{1}^{2}
$$

Hence by the hypothesis that $\Sigma \mu_{i}\left(\pi_{i} \otimes_{Z_{i}} \circ \circ \pi_{i} \circ j\left(\left|h_{i} j\left(h_{i}^{-1}\right)-1\right|_{1}^{2}\right)\right)<\infty$,

$$
\sum_{i} \sum_{|m-n|>1} \mu_{i}\left(\pi_{i} \otimes_{Z_{i}} j \circ \pi_{i} \circ j\left(e_{i}^{m} j\left(e_{i}^{n}\right)\right)\right)<\infty .
$$

This may be rewritten as

$$
\sum_{i} \sum_{|m-n|>1} \mu_{i}\left(\pi_{i}\left(e_{i}^{m}\right) \pi_{i}\left(e_{i}^{n}\right)\right)<\infty .
$$

Since $\Sigma_{m, n} \mu_{i}\left(\pi_{i}\left(e_{i}^{m}\right) \pi_{i}\left(e_{i}^{n}\right)\right)=1$, it follows that

$$
\sum_{i} \mu_{i}\left(1-\sum_{m} \pi_{i}\left(e_{i}^{m}\right) \pi_{i}\left(e_{i}^{m-1}+e_{i}^{m}+e_{i}^{m+1}\right)\right)<\infty .
$$

For each $i$ denote by $1-p_{i}$ the largest projection in $Z_{i}\left(=A_{i} \cap A_{i}^{\prime}\right)$ such that $\left(1-p_{i}\right) \pi_{i}\left(e_{i}^{m}\right) \leqslant 1 / 4$, all $m \in \mathbf{Z}$. Then for each $i$,

$$
\begin{aligned}
\sum_{m}\left(1-p_{i}\right) \pi_{i}\left(e_{i}^{m}\right) \pi_{i}\left(e_{i}^{m-1}+e_{i}^{m}+e_{i}^{m+1}\right) & \leqslant \frac{3}{4} \sum_{m}\left(1-p_{i}\right) \pi_{i}\left(e_{i}^{m}\right)=\frac{3}{4}\left(1-p_{i}\right) ; \\
\sum_{m} \mu_{i}\left(\pi_{i}\left(e_{i}^{m}\right) \pi_{i}\left(e_{i}^{m-1}+e_{i}^{m}+e_{i}^{m+1}\right)\right) & \leqslant \frac{3}{4} \mu_{i}\left(1-p_{i}\right)+\mu_{i}\left(p_{i}\right) \leqslant 1
\end{aligned}
$$

Hence

$$
\sum\left(1-\left(3 \mu_{i}\left(1-p_{i}\right) / 4+\mu_{i}\left(p_{i}\right)\right)\right)<\infty
$$

that is,

$$
\frac{1}{4} \sum \mu_{i}\left(1-p_{i}\right)<\infty
$$

In other words, if a finite number of indices $i$ are neglected then the tensor product projection $\otimes p_{i}$ is nonzero. Hence, replacing $A_{i}$ by $p_{i} A_{i} p_{i}, \mu_{i}$ by $\mu_{i}\left(p_{i}\right)^{-1} \mu_{i} \mid p_{i} A_{i} p_{i}$, and $A$ by $\left(\otimes p_{i}\right) A\left(\otimes p_{i}\right)$, we see that it is enough to prove the lemma assuming that each $p_{i}$ is 1 .

Under this assumption, there exists for each fixed $i$ a family of projections 
$\left(q_{k}\right)$ in $Z_{i}$ with sum 1 and for each $k$ an integer $m(k)$ such that $\pi_{i}\left(\Sigma_{k} q_{k} e_{i}^{m(k)}\right) \geqslant$ 1/4. For each $n \in Z$ set $\Sigma_{k} q_{k} e_{i}^{m(k)+n}=f_{i}^{n}$. Then

$$
\sum_{|n|>1} f_{i}^{0} j\left(f_{i}^{n}\right) \leqslant\left|h_{i} j\left(h_{i}^{-1}\right)-1\right|_{1}^{2} .
$$

Hence as in the second paragraph,

$$
\sum_{i} \sum_{|n|>1} \mu_{i}\left(\pi_{i}\left(f_{i}^{0}\right) \pi_{i}\left(f_{i}^{n}\right)\right)<\infty,
$$

whence by the property $\pi_{i}\left(f_{i}^{0}\right) \geqslant 1 / 4$,

$$
\sum_{i} \sum_{|n|>1} \mu_{i}\left(f_{i}^{n}\right) \leqslant 4 \sum_{i} \sum_{|n|>1} \mu_{i}\left(\pi_{i}\left(f_{i}^{0}\right) \pi_{i}\left(f_{i}^{n}\right)\right)<\infty
$$

It follows that if we set $f_{i}^{-1}+f_{i}^{0}+f_{i}^{1}=e_{i}$, then the $e_{i}$ satisfy the requirements of the conclusion of the lemma.

3.8. Lemma. Let $A=\Theta\left(A_{i}, \mu_{i}\right)$. Assume that the hypotheses of 3.6 are satisfied. Suppose that the second of the equivalent conditions of 3.6 is satisfied. Suppose that for each $i, h_{i} j\left(h_{i}^{-1}\right) \leqslant 8$. Then $A$ is finite.

Proof. From $h_{i} j\left(h_{i}^{-1}\right) \leqslant 8$ follows $\left|h_{i} j\left(h_{i}^{-1}\right)-1\right| \leqslant 7$. Therefore by the second of the equivalent conditions of 3.6, with $c=49$,

$$
\sum \mu_{i}\left(\pi_{i} \otimes_{z_{i}} j \circ \pi_{i} \circ j\left(\left(h_{i} j\left(h_{i}^{-1}\right)-1\right)^{2}\right)\right)<\infty .
$$

With $x \in A_{i}$, we calculate as follows:

$$
\begin{aligned}
\mu_{i}\left(\pi_{i} \otimes_{z_{i}} j \circ \pi_{i} \circ j\left(x j\left(h_{i}^{-1}\right)\right)\right) & =\mu_{i}\left(\pi_{i}(x) \pi_{i}\left(h_{i}^{-1}\right)\right) \\
& =\mu_{i}\left(\pi_{i}\left(\pi_{i}(x) h_{i}^{-1}\right)\right)=\mu_{i}\left(\pi_{i}(x) h_{i}^{-1}\right) \\
& =\tau_{i}\left(\pi_{i}(x)\right)=\mu_{i}\left(\pi_{i}(x)\right)=\mu_{i}(x) .
\end{aligned}
$$

We make use of this with $h_{i}$ and $h_{i}^{2}$ in place of $x$ to obtain

$$
\mu_{i}\left(\pi_{i} \otimes_{Z_{i}} j \circ \pi_{i} \circ j\left(\left(h_{i} j\left(h_{i}^{-1}\right)-h_{i}\right)\left(h_{i}-1\right)\right)\right)=0,
$$

from which follows, by the Pythagorean equation,

$$
\mu_{i}\left(\pi_{i} \otimes_{z_{i}} j \circ \pi_{i} \circ j\left(\left(h_{i} j\left(h_{i}^{-1}\right)-1\right)^{2}\right)\right) \geqslant \mu_{i}\left(\pi_{i} \otimes_{z_{i}} j \circ \pi_{i} \circ j\left(\left(h_{i} j\left(h_{i}^{-1}\right)-h_{i}\right)^{2}\right)\right) \text {. }
$$

We conclude that

$$
\sum \mu_{i}\left(\pi_{i} \otimes_{z_{i}} j \circ \pi_{i} \circ j\left(\left(h_{i} j\left(h_{i}^{-1}\right)-h_{i}\right)^{2}\right)\right)<\infty .
$$

Let us now show that $h_{i} \geqslant 1 / 8$. It follows from the fact that $\tau_{i} \mid Z_{i}$ is equal to $\mu_{i} \mid Z_{i}$ that $\tau_{i}\left(p h_{i}\right)=\tau_{i}(p)$ for any central projection $p$ in $A_{i}$. Hence, if 
$p h_{i} \leqslant 1$ for such a $p$, then $p h_{i}=1$, so there is a spectral projection $f_{i}$ of $h_{i}$ with central support 1 such that $f_{i} h_{i} \geqslant f_{i}$. The inequality $h_{i} \geqslant 1 / 8$ then follows from the condition $h_{i} j\left(h_{i}^{-1}\right) \leqslant 8$.

Since $h_{i} j\left(h_{i}^{-1}\right)-h_{i}=h_{i}\left(j\left(h_{i}^{-1}\right)-1\right)$, we now have

$$
\sum \mu_{i}\left(\pi_{i} \otimes_{z_{i}}{ } \circ \pi_{i} \circ j\left(\left(j\left(h_{i}^{-1}\right)-1\right)^{2}\right)\right)<\infty ;
$$

that is, $\Sigma \mu_{i}\left(\left(1-h_{i}^{-1}\right)^{2}\right)<\infty$. Since $\left(1-h_{i}^{-1 / 2}\right)^{2} \leqslant\left(1-h_{i}^{-1}\right)^{2}$, we have $\Sigma \mu_{i}\left(\left(1-h_{i}^{-1 / 2}\right)^{2}\right)<\infty$.

Since $\mu_{i}\left(h_{i}^{-1}\right)=\tau_{i}(1)=1$ for each $i$, it follows that the tensor product vector $\otimes h_{i}^{-1 / 2}$ exists in the tensor product Hilbert space $\otimes\left(H_{\mu_{i}}, 1\right)$, where $H_{\mu_{i}}$ is the completion of $A_{i}$ in the inner product defined by $\mu_{i}$, so that the element 1 of $A_{i}$ is also an element of $H_{\mu_{i}}$. Since this tensor product Hilbert space is also the Hilbert space $H_{\mu}$, where $\mu=\otimes \mu_{i}$, it follows that the vector $\otimes h_{i}^{-1 / 2}$ defines a state on $A$. Since this state is a normal tensor product state which on each $A_{i} \otimes 1$ agrees with $\tau_{i} \otimes 1$, it is a faithful normal trace, and $A$ is finite.

4. The type classification of tensor products of von Neumann algebras.

4.1. Comments. The type classification of tensor products of finite families of von Neumann algebras, in terms of the types of the component algebras, is now well understood-the last step was taken by Sakai, who in [8] showed that the largest semifinite projection in a tensor product (of a finite family) is the tensor product of the largest semifinite projections in the component algebras. (The same statement is also true with "semifinite" replaced by "finite", or "discrete".)

The classification into types of tensor products of infinite families of von Neumann algebras is not this straightforward, but a simple consequence of Sakai's result (together with a "zero-one" law) is that the largest senifinite projection in a tensor product of an arbitrary family of von Neumann algebras is either the tensor product of the largest semifinite projections in the component algebras or is zero. (The same situation also holds with "semifinite" replaced by "finite" or "discrete".) It is therefore sufficient just to describe conditions for a tensor product of semifinite (resp. finite, discrete) von Neumann algebras to be again semifinite (resp. finite, discrete).

For factors the type classification problem has already been almost completely solved. Let us collate Theorems $4.2,4.4$ and 4.5 below with the literature.

Condition 4.5(ii)' for a tensor product of discrete von Neumann algebras to be discrete is, in the factor case, due to Bures (it is the condition in Proposition 5.3 of [1]).

Condition 4.4(ii) for a tensor product of finite von Neumann algebras to be finite is also, in the factor case, due to Bures (it is the condition in Theorem 4.3 of [2]). Earlier work on tensor products of discrete finite factors was done by Bures [1] and Moore [7]. 
Condition 4.2(iv) for a tensor product of semifinite von Neumann algebras to be semifinite was formulated by Moore (see Lemma 3.2 of [7] and the neighbouring discussion). It is basic in the later work of Takenouchi, Hill, and Stbrmer. Moore's proof of necessity is the only one known. Sufficiency (for various classes of factors) was shown via condition 4.2(ii)" by Moore [7, Lemma 5.2], Hill [5, Theorem 3.20] and Takenouchi [10, §3]. The same route is followed here. Stbrmer [9] used an entirely different method, assuming separable predual, which is also applicable when the centre is nontrivial-see 3.4 above. Stormer's method bypasses 4.2 (ii)", but it should be noted that $4.2(\mathrm{ii})$ " is used in the proof of 4.5 (in the nonfactor case), and also to obtain 4.2 (iii).

Conditions 4.2(v) and 4.2(vi) were first formulated by Takenouchi, although related conditions had been considered by Moore in a restricted case, and the proof of the equivalence of these conditions is by means of a lemma of Moore [7, Lemma 3.5]. Takenouchi showed that 4.2(iv) implies 4.2(v), for tensor products of discrete factors $[10, \S 2]$. In [9] Stbrmer rewrote Takenouchi's proof for tensor products of continuous factors, and also proved the converse implication. Here (in 3.5) we repeat Stbrmer's proof of the converse, and reverse it to give a proof of 4.2 (iv) $\Rightarrow 4.2(\mathrm{v})$ slightly simpler than Takenouchi's. Takenouchi proved the implication $4.2(v) \Rightarrow 4.2(i i)^{\prime \prime}$. Here we show that it is easier to deduce 4.2(ii)" from 4.2(vi).

4.2. Theorem. Let $A=\bigotimes\left(A_{i}, \mu_{i}\right)$ (see 3.1). Suppose that each $A_{i}$ is semifinite. Then the following conditions are equivalent.

(i) $A$ is semifinite.

(ii) $A$ has a nonzero finite tensor product projection.

(ii)' $A$ has a finite tensor product projection of central support 1.

(ii)" $A$ has a finite tensor product projection $\otimes e_{i}$ of central support 1 such that for each $i$ the unitary $1-2 e_{i}$ is in the centre of the set of unitaries $u$ such that $u^{*} \mu_{i} u=\mu_{i}$.

(iii) $A$ has a faithful normal semifinite tensor product trace; that is, a faithful normal semifinite trace $\tau$ such that there exist faithful normal traces $\tau_{i}$ of $A_{i}$ for each $i$ with $\tau\left(\otimes a_{i}\right)=\Pi \tau_{i}\left(a_{i}\right)<\infty$ for elements $\otimes a_{i}\left(a_{i} \geqslant 0\right)$ generating a strongly dense ideal of $A$.

If each $\mu_{i}$ is faithful, then with notation as in 3.1 , these conditions are equivalent to the following.

(iv) $\Sigma \mu_{i}\left(1-\left|\pi_{i}\left(h_{i}^{i t}\right)\right|\right)<\infty$ for all real $t$.

(v) $\Sigma \mu_{i}\left(\pi_{i} \otimes_{Z_{i}} j \circ \pi_{i} \circ j\left(\left|1-\Delta_{i}^{i t}\right|^{2}\right)\right)<\infty$ for all real $t$, where $\Delta_{i}$ is the modular operator of $\mu_{i}\left(\Delta_{i}=h_{i} j\left(h_{i}^{-1}\right)\right)$.

(vi) $\Sigma \mu_{i}\left(\pi_{i} \otimes_{Z_{i}} j \circ \pi_{i} \circ j\left(\left|1-\Delta_{i}\right|_{c}^{2}\right)\right)<\infty$ for some (and hence for all) $c>0$.

Proof. (iii) $\Rightarrow$ (i) and (ii)" $\Rightarrow$ (ii) $\Rightarrow$ (ii) $\Rightarrow$ (i) are clear. (Cf. the second 
paragraph of 4.1.) (i) $\Rightarrow$ (iv) $\Longleftrightarrow$ (v) $\Longleftrightarrow$ (vi) $\Rightarrow$ (ii) was established in proving 3.2.

(i) $\Rightarrow$ (ii)". The proof of 3.2 showed that (ii)" holds with "of central support 1" replaced by "nonzero". This distinction is unimportant because of the fact that if for each $\alpha$ the tensor product $\otimes e_{i}^{\alpha}$ is finite, and if for each $i$ the $e_{i}^{\alpha}$ have mutually orthogonal central supports, then the tensor product $\otimes\left(\Sigma_{\alpha} e_{i}^{\alpha}\right)$. is finite also.

(i) $\Rightarrow$ (iii). The proof of 3.2 showed that (iii) holds with "faithful" replaced by "nonzero". The situation is closely similar to that in the preceding paragraph. (Replace $e_{i}^{\alpha}$ by $\tau_{i}^{\alpha}$, a normal semifinite trace, and in place of "finite" read "semifinite".)

4.3. REMARK. It should be noted that although condition $4.2(\mathrm{v})$ can be rewritten as $\Sigma\left|1-\bar{\mu}_{i}\left(\Delta_{i}^{i t}\right)\right|<\infty$, where $\bar{\mu}_{i}=\mu_{i} \circ\left(\pi_{i} \otimes_{Z_{i}} j \circ \pi_{i} \circ j\right)$ is a state of the $C^{*}$-algebra $B_{i}$ generated by $A_{i}$ and $A_{i}^{\prime}$, so that it is equivalent to the existence in $\otimes\left(H_{\bar{\mu}_{i}}, \xi_{\bar{\mu}_{i}}\right)$ of $\otimes \Delta_{i}^{i t}$, this condition is not the same as the existence in $\otimes\left(H_{\mu_{i}}, \xi_{\mu_{i}}\right)$ of $\otimes \Delta_{i}^{i t}$. Indeed, since $\Delta_{i}^{i t} \xi_{\mu_{i}}=\xi_{\mu_{i}}$, the latter always holds.

4.4. Theorem. Let $A=\Theta\left(A_{i}, \mu_{i}\right)$. Suppose that each $A_{i}$ is finite. For each $i$ denote by $\tau_{i}$ the unique trace of $A_{i}$ agreeing on the centre of $A_{i}$ with $\mu_{i}$. Then the following conditions are equivalent.

(i) $A$ is finite.

(ii) $\otimes \tau_{i}$ exists as a normal state of $A$. (For equivalent statements of this see $[2,4.1]$.)

Proof. (ii) $\Rightarrow$ (i). (ii) implies that $A$ has a nonzero finite central projection, whence the largest finite central projection in $A$ is 1 .

(i) $\Rightarrow$ (ii). If $A$ is finite then there exists a centre-valued trace on $A$ fixing each element of the centre. Denote this centre-valued trace by $\theta$; then $\left(\otimes \mu_{i}\right) \circ$ $\theta=\otimes \tau_{i}$.

4.5. TheOREM. Let $A=\bigotimes\left(A_{i}, \mu_{i}\right)$. Suppose that each $A_{i}$ is discrete. Then the following conditions are equivalent.

(i) $A$ is discrete.

(ii) $A$ has a nonzero abelian tensor product projection.

(ii)' $A$ has an abelian tensor product projection $\otimes e_{i}$ of central support 1 , such that for all but finitely many $i, e_{i}$ satisfies the condition stated in 4.2(ii)" (which may be described as belonging to the centre of the invariance algebra of $\left.\mu_{i}\right)$.

Proof. (ii) $\Rightarrow$ (ii) $\Rightarrow$ (i) is clear. (Cf. the second paragraph of 4.1.)

(i) $\Rightarrow$ (ii)' . By (i) $\Rightarrow$ (ii)" of 4.2 there exists a finite tensor product projection $\otimes f_{i}$ of central support 1 such that for each $i, f_{i}$ belongs to the centre of the invariance algebra of $\mu_{i}$. Then $\left(\otimes f_{i}\right) A\left(\otimes f_{i}\right)$ is both finite and discrete, and hence 
all except finitely many $f_{i}$ must be abelian. If $f_{i}$ is abelian set $f_{i}=e_{i}$. If $f_{i}$ is not abelian choose any abelian projection in $A_{i}$ of central support 1 and denote this by $e_{i}$. The $e_{i}$ then satisfy the requirements of (ii)' .

\section{REFERENCES}

1. D. J. C. Bures, Certain factors constructed as infinite tensor products, Compositio Math. 15 (1963), 169-191. MR 27 \#1809.

2. An extension of Kakutani's theorem on infinite product measures to the tensor product of semifinite $w^{*}$-algebras, Trans. Amer. Math. Soc. 135 (1969), 199-212. MR 38 \#5014.

3. A. Connes, Une classification des facteurs de type III, Ann. Sci. École Norm. Sup. 6 (1973), 133-252. \#A2066.

4. J. G. Glimm, Type I C*-algebras, Ann. of Math. (2) 73 (1961), 572-612. MR 23

5. D. G. B. Hill, o.finite invariant measures on infinite product spaces, Trans. Amer. Math Soc. 153 (1971), 347-370. MR 43 \#485.

6. R. R. Kallman, Groups of inner automorphisms of von Neumann algebras, J. Functional Analysis 7 (1971), 43-60. MR 43 \#5317.

7. C. C. Moore, Invariant measures on product spaces, Proc. Fifth Berkeley Sympos. Math. Statist. and Probability (Berkeley, Calif., 1965/66), Vol. II: Contributions to Probability Theory, part 2, Univ. of California Press, Berkeley, Calif., 1967, pp. 447-459. MR 37 \#2951.

8. S. Sakai, On topological properties of $W^{*}$-algebras, Proc. Japan Acad. 33 (1957), 439-444. MR 20 \#5437.

9. E. Stprmer, On infinite tensor products of von Neumann algebras, Amer. J. Math. 93 (1971), 810-818. MR 44 \#5792.

10. O. Takenouchi, On type classification of factors constructed as infinite tensor products, Publ. Res. Inst. Math. Sci. Ser. A 4 (1968/69), 467-482. MR 40 \#762.

11. M. Takesaki, On the cross-norm of the direct product of $C^{*}$-algebras, Tôhoku Math. J. (2) 16 (1964), 111-122.

12. Tomita's theory of modular Hilbert algebras and its applications, Lecture Notes in Math., vol. 128, Springer-Verlag, New York, 1970.

SCHOOL OF MATHEMATICS, INSTITUTE FOR ADVANCED STUDY, PRINCETON, NEW JERSEY 08540 DENMARK

MATHEMATICS INSTITUTE, UNIVERSITY OF COPENHAGEN, COPENHAGEN, 